\title{
Laterally Coupled-Cavity Semiconductor Lasers
}

\author{
ROBERT J. LANG, MEMBER, IEEE, AMNON YARIV, FELLOW, IEEE, AND JOSEPH SALZMAN
}

\begin{abstract}
We analyze the threshold behavior of a pair of laterally coupled semiconductor lasers of different lengths. The predictions include longitudinal mode selectivity leading to single longitudinal mode operation with a periodicity determined by the length mismatch, and ripples in the equipower curves in the current plane due to carrierinduced index shifts. We present experimental measurements that confirm these predictions.
\end{abstract}

\section{INTRODUCTION}

$\mathrm{T}$ HE desire for narrow linewidths in lasers used in fiber optic transmission systems has made longitudinal mode control the subject of study because single-mode lasers are less noisy than their multimode counterparts. The latter are plagued by partition noise resulting from competition among the modes. Unfortunately, the most common laser geometry - a longitudinally homogeneous waveguide bounded by two flat mirrors-usually runs in multiple longitudinal modes due to gain saturation and high spontaneous emission.

More complicated laser structures have been proposed that discriminate between longitudinal modes. They include distributed feedback lasers [1] and distributed Bragg reflectors [2], which contain a corrugated grating with a period of half the optical wavelength. DFB's and DBR's suffer from difficulties in fabrication due to the need to bury a very fine structure underneath the upper cladding layers without introducing defects into the crystal structure. Another direction of research has encompassed coupled-cavity lasers [3], [4] in which multiple Fabry-Perot resonators are coupled together. They are of particular interest because they are relatively simple to fabricate and offer the potential of FM operation [5], linewidth reduction, and modulation speed enhancement [6]-[8], along with single-mode operation.

To date, the most common geometry of coupled-cavity lasers has been longitudinal, that is, the two lasers are butted up against each other end to end. In this geometry, the gap between the two lasers plays a crucial role in the laser operation. For best gain selectivity, it must be a (small) integral number of half wavelengths [9], [10]. Unfortunately, accurate control over the gap requires mechanical adjustment, which is undesirable in a system.

An alternative is to monolithically fabricate two lasers

Manuscript received July 28,1986 ; revised November 21,1986 . This work was supported by the National Science Foundation and the Office of Naval Research.

R. J. Lang and A. Yariv are with the California Institute of Technology, Pasadena, CA 91125.

J. Salzman is with Bell Communications Research, Red Bank, NJ 07701. IEEE Log Number 8613068.

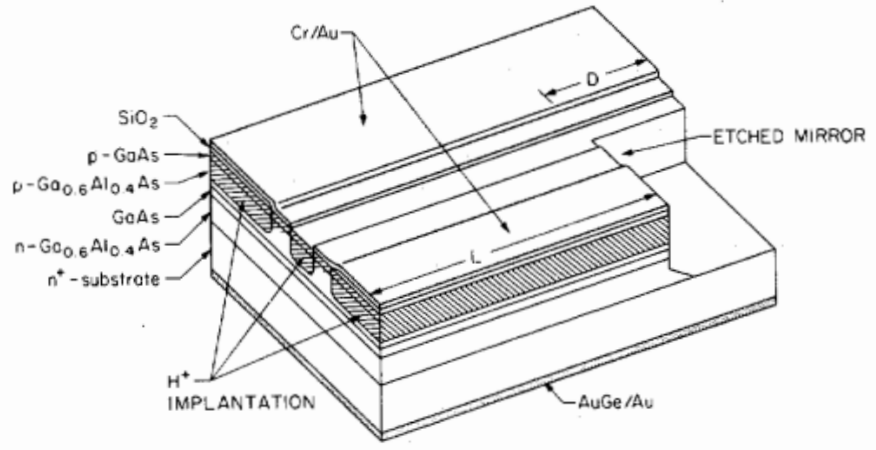

Fig. 1. Schematic drawing of a laterally coupled-cavity semiconductor laser.

side by side so that the coupling occurs via the evanescent fields of the individual lasers [11], [12]. If the lasers are of different lengths, then the longitudinal spectra of the two lasers differ, and we expect low thresholds only where the longitudinal modes of the two lasers coincide. In this paper, we present a theory of, and experimental measurements on, a laterally coupled-cavity laser. In Section II, we outline the theory of operation and calculate some representative threshold gain curves that illustrate the gain discrimination. In Section III, we present experimental measurements on the device. In Section IV, we summarize the important points of the paper.

\section{THEORY OF OpERATION}

The device under consideration is illustrated in Fig. 1. It consists of two lasers of length $L_{1}$ (short) and $L_{2}$ (long), characterized by propagation constants $\beta_{1}$ and $\beta_{2}$, respectively. We seek to understand the modes of such a structure and to calculate the threshold gains of each mode. Our intuition suggests the following: each cavity is on resonance when the optical path length seen by a field as it completes a round trip of the cavity becomes an integral number of wavelengths. Only for a few select frequencies will this condition be satisfied simultaneously in both cavities. However, the situation is more complicated than that. In the region of the laser where the two lasers are side by side, a field cannot propagate in one cavity alone, due to the coupling between the two cavities. The appropriate description of the system is in terms of the supermodes [12], that is, the modes of the twin waveguide.

Any field at a fixed position in the cavity can be written either as a sum of the supermodes of the cavity or as a sum of the modes of the individual channels. Along the laser length, there are portions of a single waveguide and 
portions where the two waveguides are coupled, so we need a means to switch from the supermode representation (SM) to a channel mode $(\mathrm{CM})$ representation. We first define the propagation constants of the isolated channels.

$$
\beta_{1,2} \equiv \frac{\omega \mu_{0}}{c}+(\alpha+j) \frac{\gamma_{1,2}}{2}
$$

where the subscript 1,2 refers to the channel, $\omega$ is the lasing frequency, $\mu_{0}$ is the nonresonant refractive index, $\gamma_{1,2}$ is the gain in each channel, and $\alpha$ is the linewidth enhancement factor relating changes in the real and imaginary index of refraction. When the two cavities are coupled, standard coupled-mode theory gives a good approximation to the propagation constants and fields by assuming that the supermodes are composed of a linear combination of the channel modes. We define coupling coefficients as overlap integrals of the channel mode fields:

$$
\begin{aligned}
\kappa_{12} & \equiv \int \Delta \mu_{1}^{2} E_{1}(x) E_{2}(x) d x, \\
\kappa_{21} & \equiv \int \Delta \mu_{2}^{2} E_{2}(x) E_{1}(x) d x
\end{aligned}
$$

where $\Delta \mu_{1,2}$ is the perturbation in index seen by one channel mode due to the other channel. Then, if we make the definitions

$$
\begin{gathered}
\bar{\beta} \equiv \frac{\beta_{1}+\beta_{2}}{2}, \quad \Delta \beta \equiv \frac{\beta_{1}-\beta_{2}}{2}, \\
S \equiv \sqrt{\kappa_{12} \kappa_{21}+\Delta \beta^{2}},
\end{gathered}
$$

the propagation constants of the two supermodes are given by

$$
\sigma_{1,2}=\bar{\beta} \pm S
$$

Any field that is represented by a linear sum of the channel mode fields can be written as a linear sum of the supermode fields as well. If we represent a field by a vector $\underline{A} \equiv\left(\begin{array}{ll}a_{1} & a_{2}\end{array}\right)_{C M}$ where $a_{1}$ and $a_{2}$ are the amplitudes of the two channel modes; then the amplitudes $b_{1}$ and $b_{2}$ of the two supermodes describing the same field can be written as

$$
\underline{B} \equiv\left(\begin{array}{l}
b_{1} \\
p_{2}
\end{array}\right)_{S M}=\underline{\underline{V}}\left(\begin{array}{l}
a_{1} \\
a_{2}
\end{array}\right)_{C M}
$$

The square matrix $\underline{\underline{V}}$ is given by

$$
\underline{\underline{V}} \equiv\left(\begin{array}{cc}
p_{1} & -p_{2} \\
p_{2} & p_{1}
\end{array}\right)
$$

with $p_{1,2}^{2} \equiv(1 \pm \Delta \beta / S) / 2$. We point out that $\underline{\underline{V}}$ is unitary, that is, $\underline{\underline{V}}^{-1}=\underline{\underline{V}}^{T}$.

Obviously, $\underline{\bar{A}}=\underline{\underline{V}}^{\overline{-1}} \underline{B}$. We can also write the effects of any linear operation upon the fields as a square matrix that is unique within a given representation. For example, in the channel mode representation, the field after an encounter with a mirror of reflectivity $r_{0}$ would be

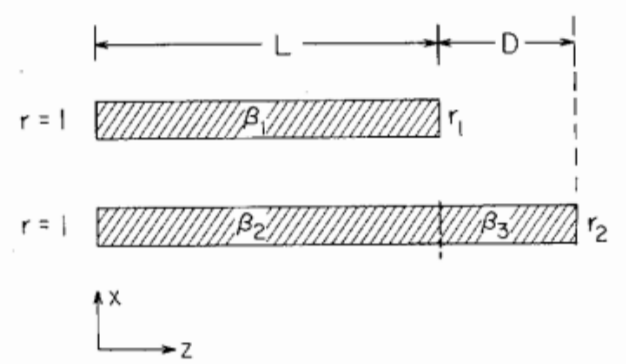

Fig. 2. Schematic representation of an idealized laterally coupled-cavity laser.

$$
\underline{A}_{\text {after }}=\left(\begin{array}{cc}
r_{0} & 0 \\
0 & r_{0}
\end{array}\right) \underline{A}_{\text {before }} .
$$

However, to write the appropriate operator for a supermode vector, we must transform the operator to the new representation. We accomplish this with the matrix $\underline{V}$. For an operator $T$, if we denote the channel representation of this operator by $\underline{\underline{T}}_{C M}$ and the supermode representation by $\underline{\underline{T}}_{S M}$, the two matrices are related by

$$
\underline{\underline{T}}_{S M}=\underline{\underline{V}} \underline{\underline{T}} C M \underline{\underline{V}}^{-1}, \quad \underline{\underline{T}} C M=\underline{\underline{V}}^{-1} \underline{\underline{T}}_{S M} \underline{\underline{V}} .
$$

The need for switching between representations arises because the matrices for some operations (reflection, propagation) assume a simpler form in one representation than the other. Let us choose an arbitrary field in the supermode representation $\underline{B}_{S M}$ at $z=0$ in Fig. 2, and calculate the matrix that propagates it through one round trip of the resonator. We do this by composing a matrix for each portion of the journey and appending it to the left side of the initial matrix, the identity matrix [14], [15].

We begin by propagating from $z=0$ to $z=L$. That matrix, in the supermode representation, is given by inspection. It is

$$
\underline{\underline{P}}_{S M} \equiv\left(\begin{array}{ll}
e^{-j \sigma_{1} L} & 0 \\
0 & e^{-j \sigma_{2} L}
\end{array}\right)
$$

since each supermode merely gains a phase factor. At $L$, we must switch over to a channel mode representation by multiplying by a factor $V^{-1}$. The field in channel 1 sees a reflectivity $r_{1}$, while the field in channel 2 propagates further for a distance $D$, gets reflected by reflectivity $r_{2}$, and then propagates back to $z=L$. This matrix can be written as

$$
\underline{\underline{R}}_{C M}^{R}=\left(\begin{array}{ll}
r_{1} & 0 \\
0 & r_{2} e^{-2 j \beta_{2} D}
\end{array}\right) .
$$

Now we transfer back to the supermode representation by multiplying by $\underline{\underline{V}}$. We propagate back to $z=0$ with the matrix $\underline{\underline{P}}_{S M}$, and reflect off the left mirror. For the case of uniform reflectivity on the left, the reflection matrix takes the same form in either representation:

$$
\underline{\underline{R}}_{C M}^{L}=\underline{\underline{R}}_{S M}^{L}=\left(\begin{array}{cc}
r_{0} & 0 \\
0 & r_{0}
\end{array}\right)
$$




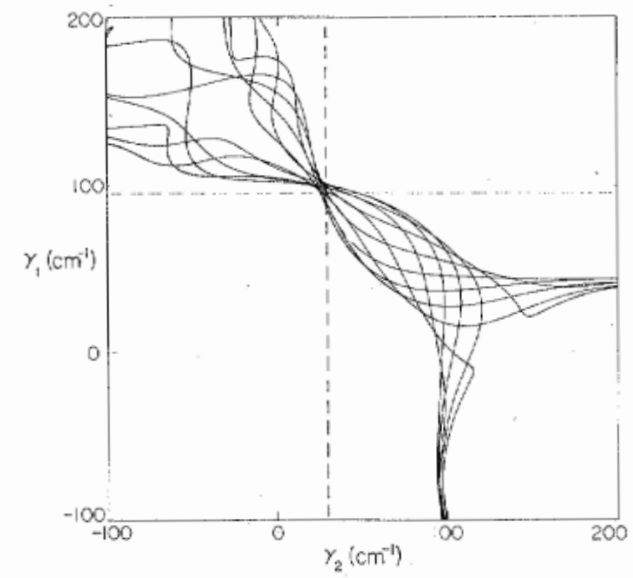

Fig. 3. Threshold gain in the $\left(\gamma_{1}, \gamma_{2}\right)$ plane for an $\mathrm{LC}^{2}$ laser consisting of two segments of lengths 200 and $240 \mu \mathrm{m}$ and intercavity coupling coefficient $K=10 \mathrm{~cm}^{-1}$. Dashed lines indicate threshold gains of the individual lasers in the absence of coupling.

So, our round-trip matrix is given by

$$
\underline{\underline{T}}_{S M}=\underline{\underline{\underline{R}}}_{S M}^{L} \underline{\underline{P}}_{S M} \underline{\underline{V}}_{\underline{\underline{R}}}^{R} \underline{\underline{R}}^{\underline{\underline{V}}} \underline{\underline{\underline{P}}}^{-1} \underline{\underline{P}}_{S M}
$$

The lasing condition that a field $\underline{B}_{S M}$ reproduces itself exactly after one round trip can be expressed as

$$
\underline{\underline{T}}_{S M} \underline{B}_{S M}=\underline{B}_{S M}
$$

We recognize this as an eigenvalue problem. The matrix $\underline{\underline{T}}_{S M}-\underline{I}$ must be singular to have a nontrivial solution for $\underline{\underline{\bar{B}}}_{S M}$. If we define

$$
\begin{aligned}
r_{1 e f f} & \equiv p_{1}^{2} r_{1}+p_{2}^{2} r_{2} e^{-2 j \beta_{2} D} \\
r_{2 e f f} & \equiv p_{2}^{2} r_{1}+p_{1}^{2} r_{2} e^{-2 j \beta_{2} D} \\
r_{\Delta} & \equiv p_{1} p_{2}\left(r_{1}-r_{2} e^{-2 j \beta_{2} D}\right),
\end{aligned}
$$

the associated secular equation can be written as

$$
\begin{gathered}
{\left[r_{1 e f f} e^{-2 j \sigma_{1} L}-1\right]\left[r_{2 e f f} e^{-2 j \sigma_{2} L}-1\right]} \\
=r_{\Delta}^{2} e^{-2 j\left(\sigma_{1}+\sigma_{2}\right) L} .
\end{gathered}
$$

The roots to (15) implicitly define the threshold gains $\gamma_{1,2}$ and lasing frequency $\omega$ of the longitudinal modes because the propagation constants $\beta_{1,2}$, and thus the supermode propagation constants $\sigma_{1,2}$, are functions of gain and frequency.

In Fig. 3, we have plotted the threshold gains for 11 adjacent longitudinal modes of a representative $L^{2} C^{2}$ laser consisting of two phase-matched $\left(\left|\beta_{1}-\beta_{2}\right|^{2}<<\left|\kappa_{1} \kappa_{2}\right|\right)$ channels of lengths 200 and $240 \mu \mathrm{m}$ (for this set of cavity lengths, the longitudinal mode spectrum possesses 11-fold periodicity). The mirror reflectivities of the two cavities were taken to be 0.55 and 0.1 , respectively (the former number is the dielectric reflectivity of the GaAs/air interface; the latter reflects imperfections in the etched mirror [16]). On the same graph, we have plotted the threshold gains for the modes when the coupling disappears (independent lasers or phase-mismatched channels). When the channels are mismatched, the supermodes are localized on one channel or the other; consequently, the longitudinal modes of the resonator are just the longitudinal modes of the individual cavities and are degenerate. Thus, the horizontal and vertical dashed lines in Fig. 2 correspond to 6 and 5 modes, respectively. Where the two lines cross, all 11 modes are degenerate.

There are several features of interest to be gleaned from this graph. The first is the broken degeneracy of the longitudinal modes, as seen by the spread curves in Fig. 3 . Fig. 3 shows a two-dimensional space of potential operating points; the only accessible region of the $\gamma_{1}-\gamma_{2}$ plane for steady-state operation is the region below and to the left of all of the curves in the graph (corresponding to subthreshold operation) and the locus of sections of threshold curves that makes up the boundary of that region (corresponding to laser operation). (The reasons for this restriction are discussed in somewhat more detail in [17].) The gain differences between adjacent modes is related to the spacing between the first mode to lase (the first line encountered as one moves out from the origin) and subsequent modes. This spacing is shaded in Fig. 3. The wider the shaded region is, the greater the gain separation at the adjacent operating point is. We see that the greatest spacing and the greatest mode discrimination arises when $\gamma_{2}$ is large and $\gamma_{1}$ is small or when we pump the lossy laser hard. The modes become nearly degenerate when both lasers are brought close to threshold. This mode of operation is roughly analogous to the situation in axially coupled lasers when the "gap" is an odd number of quarter wavelengths [18]. In our case, the equivalent parameter $\left(r_{\Delta}\right)$ varies its phase with current. So, for example, the "gap" could be adjusted by adding an independent third contact to the additional section of laser that acts as a tuning stub. Another feature to observe is that the plot of $\gamma_{1 \text { th }}$ versus $\gamma_{2 \text { th }}$ contains several ripples due to the changes in optical path length with gain via the $\alpha$ parameter (taken to be -5 for the plots). Finally, we see from the formulas that the distance that controls the periodicity of the structure is $D$, the difference in path length.

\section{Experimental Measurements}

The devices were fabricated upon GaAlAs double heterostructures grown by liquid phase epitaxy. Twin gain stripes $4 \mu \mathrm{m}$ wide with center-to-center separations of 9 $\mu \mathrm{m}$ were defined by proton implantation at $70 \mathrm{keV}$. CrAu contacts were evaporated on the surface, and the mirror of the shorter laser was etched using techniques similar to those described in [16]. The devices were lapped down to 75-100 $\mu \mathrm{m}$ thickness and AuGe contacts were evaporated on the bottom and annealed under $\mathrm{H}_{2}$ at $380^{\circ}$ for $20 \mathrm{~s}$. The devices were then cleaved into varying lengths with varying differences in cavity length.

One feature that became apparent immediately was that nearly equal cavity lengths were better for getting singlemode operation. As the model suggests, the difference in cavity lengths determines the periodicity of the longitudinal mode spectrum. The spectrum of a device with a fairly long difference is shown in Fig. 4, with a sinusoid of period $c / 2 \mu D$ superimposed over it. Also shown is the spectrum of the two devices when operated indepen- 


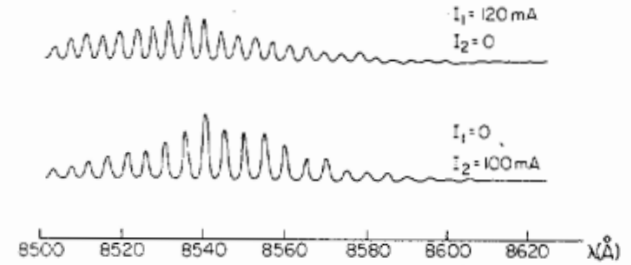

(a)

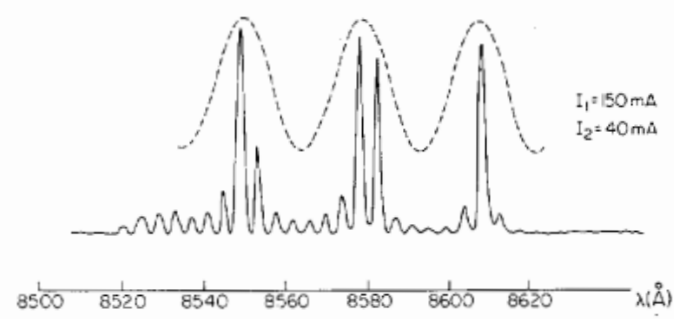

(b)

Fig. 4. Longitudinal mode spectrum for a laser of length $L=450 \mu \mathrm{m}$, path difference $D=60 \mu \mathrm{m}$. (a) Spectrum when lasers are operated separately. (b) Spectrum of the composite structure with the periodicity of the gap superimposed.

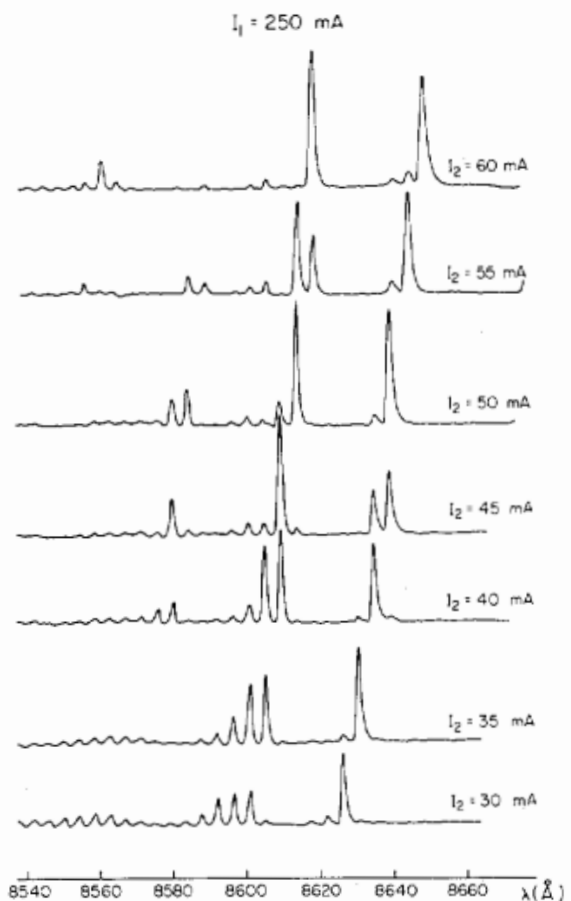

Fig. 5. Longitudinal mode spectrum of the device of Fig. 4 as a function of tuning current in cavity 2 (the long cavity).

dently. This shows another feature that is common to coupled cavities, but that has not been adequately explained; when two or more cavities are coupled together to reduce the number of longitudinal modes, the gain curve (as inferred from amplitudes of the longitudinal modes) appears to shift to a longer wavelength. One possible explanation is that the losses of coupled-cavity geometries that have reported this phenomenon (see, for example, [19]) are larger than in the uncoupled case (see Fig. 3), and the increased loss necessitates harder pumping and shifts the gain curve. The shift in optical path length with carrier density can be seen in Fig. 5 where cavity number 2 is

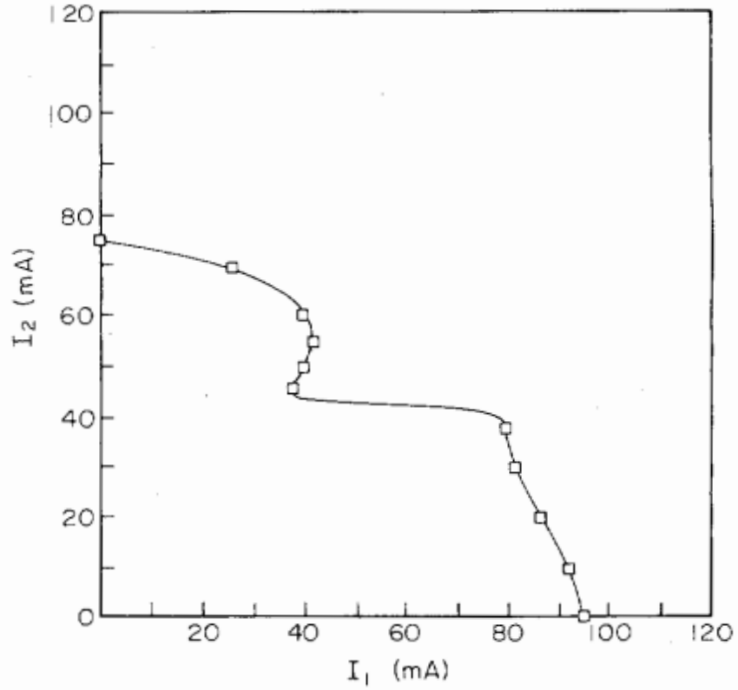

Fig. 6. Threshold gains of a device with $D \approx 3 \mu \mathrm{m}$.

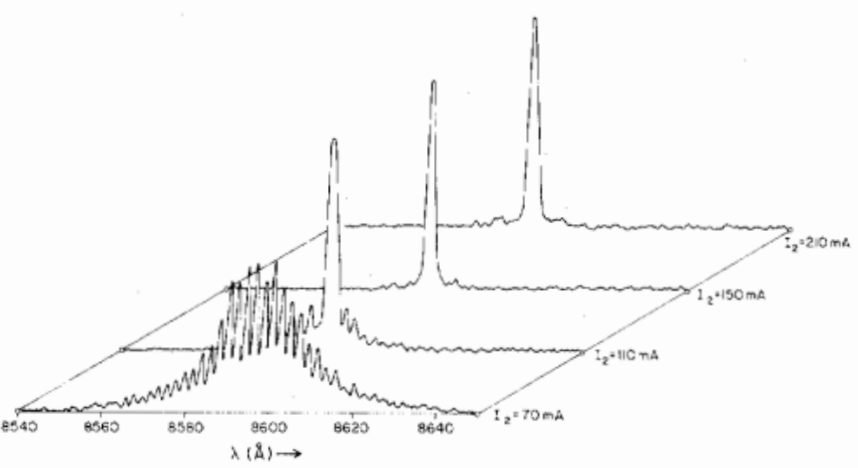

Fig. 7. Optical spectra for different currents for the device of Fig. 6 for different currents (not to the same vertical scale). The device lased in the same single longitudinal mode from threshold up to twice threshold.

pumped successively harder, thus increasing the carrier density in the additional section of length $D$ and shifting the longitudinal modes.

Smaller differences in cavity length demonstrate other phenomena. Fig. 6 shows the threshold currents (proportional to the threshold gains) required by the two cavities for a $D=3 \pm 1 \mu \mathrm{m}$ device, illustrating the ripples from interference. (For larger differences, the ripples are finer and are beyond the measurement resolution of our system.) This particular device lased in a single longitudinal mode from threshold up to a current level of twice threshold for asymmetric pumping (Fig. 7). Yet another device $(D=10 \mu \mathrm{m})$ shows single-mode operation over limited current ranges of about 20 percent of threshold, and shows a mode hop between single modes; both modes are on the long-wavelength side of the subthreshold gain curve. As before, the periodicity is controlled by the difference in cavity lengths (Fig. 8).

\section{Conclusions}

In conclusion, we have presented a device capable of single-longitudinal mode operation that is easily fabricated monolithically, a laterally coupled-cavity laser. We note that for optoelectronic integration, it will be desira- 


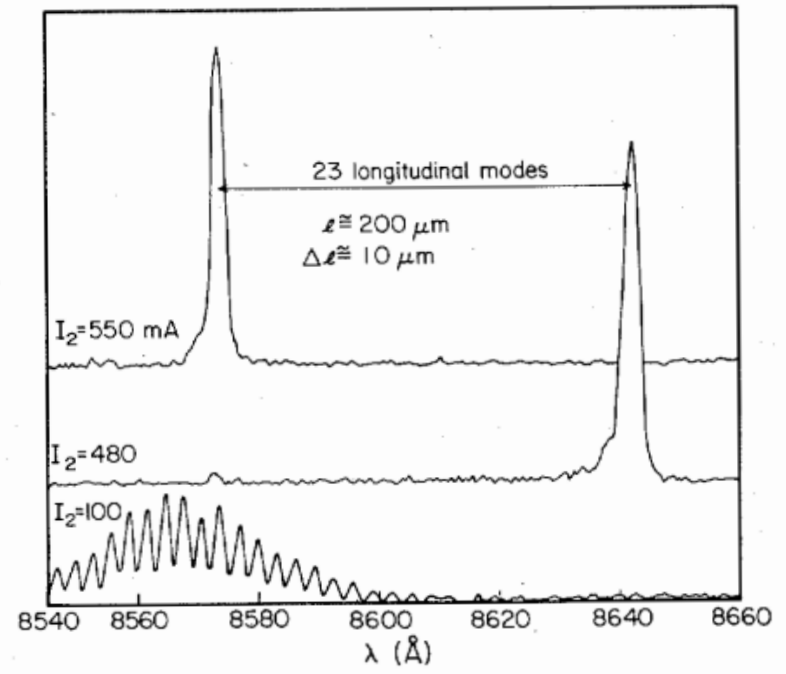

Fig. 8. Spectrum of a $D=10 \mu \mathrm{m}$ device showing a mode hop and the periodicity of the path difference.

ble to etch all mirrors of a laser, and the scheme of etching and one laser shorter than the other fits neatly within this plan. Despite the operation of this laser under pulsed conditions and the fact that the modes were gain guided (which means that the coupling coefficients change somewhat with pump current), large regimes of single-mode operation were obtained. We expect that more stable operation (allowing larger current excursions) will result from an index-guided structure where the coupling coefficients are constant and the amount of spontaneous emission is less. These results indicate that a laterally coupledcavity laser designed for $\mathrm{CW}$ operation (e.g, a twin buried heterostructure) may be suitable for use in single-mode laser systems.

\section{REFERENCES}

[1] M. Nakamura, K. Aiki, J.-I. Imeda, A. Yariv, H. W. Yen, and T. Morkawa, "GaAs-GaAlAs double heterostructure distributed feedback diode lasers,"' Appl. Phys. Lett., vol. 25, pp. 487-489, 1985.

[2] H. W. Yen, W. Ng, I. Samid, and A. Yariv, "GaAs distributed Bragg reflector lasers," Opt. Commun., vol. 17, p. 213, 1985.

[3] L. A. Coldren, B. I. Miller, K. Iga, and J. A. Rentschler, "Monolithic two-section GaInAsP/InP active-optical-resonator devices formed with RIE mirrors," Appl. Phys. Lett., vol. 38, pp. 315-317, 1981.

[4] W. T. Tsang and N. A. Olsson, "High speed direct single-frequency modulation with large tuning rate and frequency excursion in cleaved coupled cavity semiconductor lasers," Appl. Phys. Lett., vol. 42, pp. $650-652,1983$.

[5] W. Streifer, D. Yevick, T. L. Paoli, and R. D. Burnham, "Cleaved coupled cavity laser analysis," paper $\mathrm{ThO} 4$ presented at Opt. Soc. Amer. Meet., San Diego, CA, 1984.

[6] K. Vahala and A. Yariv, "Detuned loading in coupled cavity semiconductor lasers-Effect on quantum noise and dynamics," Appl. Phys. Lett., vol. 45, pp. 501-503, 1984.

[7] K. Vahala, J. Paslaski, and A. Yariv, "Observation of modulation speed enhancement, frequency modulation suppression and phase noise reduction by detuned loading in a coupled cavity semiconductor laser,"' Appl. Phys. Lett., vol. 46, pp. 1025-1027, 1985.

[8] R. J. Lang and A. Yariv, "Semiclassical theory of noise in multielement semiconductor lasers," IEEE J. Quantum Electron., vol. QE20, pp. 436-445, 1986.

[9] C. H. Henry and R. F. Kazarinov, "'Stabilization of single-frequency operation of coupled-cavity lasers," IEEE J. Quantum Electron., vol. QE-20, pp. 733-744, 1984.

[10] L. A. Coldren, T. L. Koch, C. A. Burrus, and R. G. Swartz, "In- tercavity coupling gap width dependence in coupled-cavity lasers," Electron. Lett., vol. 20, pp. 350-356, 1984.

[11] J. Salzman, R. Lang, and A. Yariv, "Laterally coupled cavity semiconductor laser," Appl. Phys. Lett., vol. 47, pp. 195-197, 1985.

[12] F. Kappeler, "Improved dynamic single-frequency low-chirp operation of a laterally coupled ridge waveguide (LCRW) laser," in Proc. Sth Int. Conf. Integrated Opt. Fiber Commun./11th European Conf. Opt. Commun., Venezia, Italy, Oct. 1985, pp. 771-774.

[13] A. Yariv, Introduction to Optical Electronics, 2nd ed. New York: Holt, Rinehart and Winston, 1976, p. 365.

[14] J. Salzman, R. Lang, and A. Yariv, "Frequency selectivity in laterally coupled semiconductor laser arrays," Opt. Lett., vol. 10, pp. 387-389, 1985.

[15] K.-L. Chen and S. Wang, "Effects of mirror imperfections on phaselocked semiconductor laser arrays," IEEE J. Quantum Electron., vol. QE-21, pp. 264-270, 1985.

[16] J. Salzman, T. Venkatesan, S. Margalit, and A. Yariv, "Etching feedback facets for GaAs heterostructure lasers," J. Appl. Phys., vol. 57, pp. 2948-2953, 1985.

[17] R. J. Lang and A. Yariv, "Intermodal stability of a coupled-cavity laser," IEEE J. Quantum Electron., vol. QE-22, pp. 631-638, 1986.

[18] L. A. Coldren and T. L. Koch, "Analysis and design of coupledcavity lasers, Part I: Threshold gain analysis and design guidelines," IEEE J. Quantum Electron., vol. QE-20, pp. 659-682, 1984.

[19] K. K. Anderson, R. H. Rediker, and L. J. Van Ruyven, "Pulsed operation of an array of diode lasers with feedback in the Fourier plane," paper ThCC3, presented at Integrated and Guided Wave Opt. Conf., Atlanta, GA, Feb. 1986.

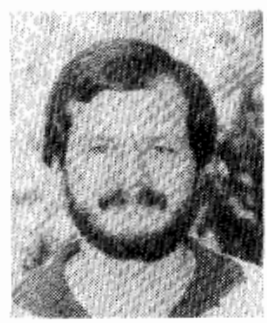

Robert J. Lang (S'83-M'86) was born in Dayton, OH, on May 4, 1961, and was raised in Atlanta, GA. In 1982, he received the B.S. degree in electrical engineering from the California Institute of Technology, Pasadena. He received the M.S. degree, also in electrical engineering, from Stanford University, Stanford, CA, in 1983. In 1986, he received the $\mathrm{Ph} . \mathrm{D}$. degree in applied physics from Caltech, for work on coupled-cavity and unstable resonator semiconductor lasers.

Currently, he is investigating dynamic and spectral properties of single-mode lasers at the Standard Elektrik Lorenz Research Centre, Stuttgart, West Germany. He has written over twenty technical papers and two books.

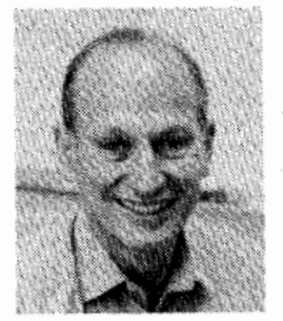

Amnon Yariv (S'56-M'59-F'70) was born in Tel Aviv, Israel, on April 13, 1930. He received the B.S., M.S., and Ph.D. degrees in electrical engineering from the University of California, Berkeley, in 1954, 1956, and 1958, respectively.

A veteran of the Israeli war of independence, he came to the United States in 1951 . He went to Bell Laboratories, Murray Hill, NJ, in 1959, joining the early stages of the laser effort. He joined the California Institute of Technology, Pasadena, in 1964, as an Associate Professor of Electrical Engineering, becoming a Professor in 1966. In 1980 he became the Thomas G. Myers Professor of Electrical Engineering and Applied Physics. On the technical side, he took part (with various co-workers) in the discovery of a number of early solid-state laser systems, in the formulation of the theory of parametric quantum noise and the prediction of parametric fluorescence, in the invention of the technique of mode-locked ultrashort-pulse lasers and FM lasers, in the introduction of GaAs and CdTe as infrared electrooptic and window materials, in proposing and demonstrating semiconductorbased integrated optics technology, and in pioneering the field of phase conjugate optics. His present research efforts are in the areas of nonlinear 
optics, recombination mechanisms in semiconductors, semiconductor lasers and integrated optics, especially the problem of monolithic integration of transistors, injection lasers, and detectors for high-frequency applications. He has authored or coauthored some 300 papers in professional journals, as well as a number of basic texts in quantum electronics, optics, and quantum mechanics. He is a Consultant to the Hughes Aircraft Research Laboratories, and is a founder and Chairman of the Board of Ortel Corporation, Alhambra, CA.

Dr. Yariv is a member of the American Physical Society, Phi Beta Kappa, and the National Academy of Engineering, and a Fellow of the Optical Society of America. He was the recipient of the 1980 Quantum Electronics Award of the IEEE. He is also an Associate Editor of Optics Communications and the Journal of Applied Physics, and was previously an Associate Editor of the IEEE JOURNAL OF QUANTUM ElECTRONICS.

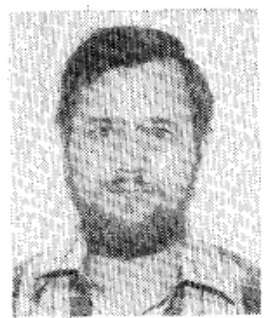

Joseph Salzman was born in Buenos Aires, $\mathrm{Ar}$ gentina on April 17, 1945. He received the B.Sc., M.Sc., and Ph.D. degrees from Tel Aviv University, Tel Aviv, Israel, 1973, 1979 and 1984, respectively.

He was nominated for a Fulbright Fellowship and the Bantrell Post-Doctoral Fellowship at the California Institute of Technology, Pasadena, where he started his work on semiconductor lasers. In March 1986 he joined Bell Communications Research, Red Bank, NJ, where he is currently working on semiconductor laser physics. His research interests include material growth, device processing and characterization of semiconductor lasers, as well as coherent communication systems. 